\title{
On the role of wave-particle interactions in the evolution of solar wind ion distribution functions
}

\author{
Lorenzo Matteini*, Simone Landi ${ }^{\dagger}$, Marco Velli**, ${ }^{*}$ and Petr Hellinger ${ }^{\ddagger}$ \\ ${ }^{*}$ LESIA, Observatoire de Paris, France \\ ${ }^{\dagger}$ Dipartimento di Astronomia e Scienza dello Spazio, Università degli Studi di Firenze, Italy \\ ** Jet Propulsion Laboratory, Pasadena, California U.S. \\ ${ }^{\ddagger}$ Institute of Atmospheric Physics and Astronomical Institute, AS CR, Prague, Czech Republic.
}

\begin{abstract}
We investigate the role of kinetic effects in the solar wind expansion using 1-D numerical hybrid simulations. The analysis of proton distribution functions in the solar wind shows a non-adiabatic evolution and suggests that several kinetic processes are at work during the expansion. From simulation studies wave-particle and wave-wave interactions, as cyclotron heating and non-linear trapping due to parametric instabilities, are found to play an important role on constraining the proton temperature anisotropy and generating secondary velocity beams. We report results from hybrid comoving simulations that self-consistently retain and describe these processes. We find that cyclotron interactions control the evolution of the proton temperature anisotropy with distance providing a perpendicular heating which contrasts the adiabatic cooling caused by the expansion. At the same time ion-acoustic modes driven by parametric effects produce a velocity beam in the proton distribution function. The resulting proton distribution functions are reasonable agreement with those observed in situ.
\end{abstract}

Keywords: Solar Wind: Plasma Simulations: Kinetic Physics, Wave-Particle Interactions

PACS: $52.35 . \mathrm{Mw}$, 52.65.Ww, 96.50.Ci, 94.05.Pt

\section{INTRODUCTION}

In situ measurements of solar wind distribution functions present departures from the local thermal equilibrium. Proton distributions often show the presence of a temperature anisotropy with respect the ambient magnetic field $T_{\perp p} \neq T_{\| p}$ and velocity beams [1]. These non-thermal properties are the signature of kinetic processes that play a role in the expansion of the solar wind plasma. In particular at scales close to the ion and electron cyclotron frequencies wave-particle interactions are likely at work, providing a continuos exchange of energy between field fluctuations and particles. These include plasma kinetic instabilities, where energy is transferred from particle to unstable modes, leading to a local wave activity generation [2]. In this case the source of free energy comes from the non-thermal properties of the plasma distribution functions, like temperature anisotropies and beams. There are indications that instabilities driven by a temperature anisotropy are at work in the slow solar wind $[3,4]$ and in the fast wind[5] at $1 \mathrm{AU}$ and beyond.

Another kind of wave-particle interaction is wave damping, corresponding to plasma heating, like ioncyclotron interaction that can provide strong perpendicular heating. The typical signature of cyclotron heating in distribution functions is the kinetic shell shape; this corresponds to a plateau in the perpendicular direction which is often observed in solar wind proton distributions [6]. Signatures of a preferential perpendicular heating of protons and alpha particles in the solar wind, as a function of their relative velocity drift has been recently discussed by [7].

Protons in fast wind streams are characterized by a typical temperature anisotropy with $T_{\perp p}>T_{\| p}$; this anisotropy seems to originate within 3 solar radii [8] in the corona, but despite the strong perpendicular cooling predicted by a radial expansion as the solar wind, it is still observed at larger heliocentric distances. Helios data between $0.3 \mathrm{AU}$ and $1 \mathrm{AU}$ show proton distribution with $T_{\perp p}>T_{\| p}$ and clearly indicate that the proton anisotropy evolves non-adiabatically [e.g. 5]. Such observations then suggest that some additional perpendicular heating contrasting the adiabatic cooling is at work along the fast wind expansion in order to maintain the observed anisotropy.

The aim of this study is to discuss the self-consistent evolution of proton distributions with increasing heliocentric distance, under the effect of the kinetic processes described above, focusing on the role of wave-particle interactions in the regulation of plasma heating and generation of velocity beams.

We perform 1-D hybrid simulations that include waveparticle and wave-wave interactions. We first present results from standard hybrid simulations, and we discuss the role of parametric instabilities on shaping the ion distribution. Then we report a simulation where the expansion effects are also taken into account and includes the evolution of a spectrum of Alfvén waves that enter in cyclotron resonance with protons. 

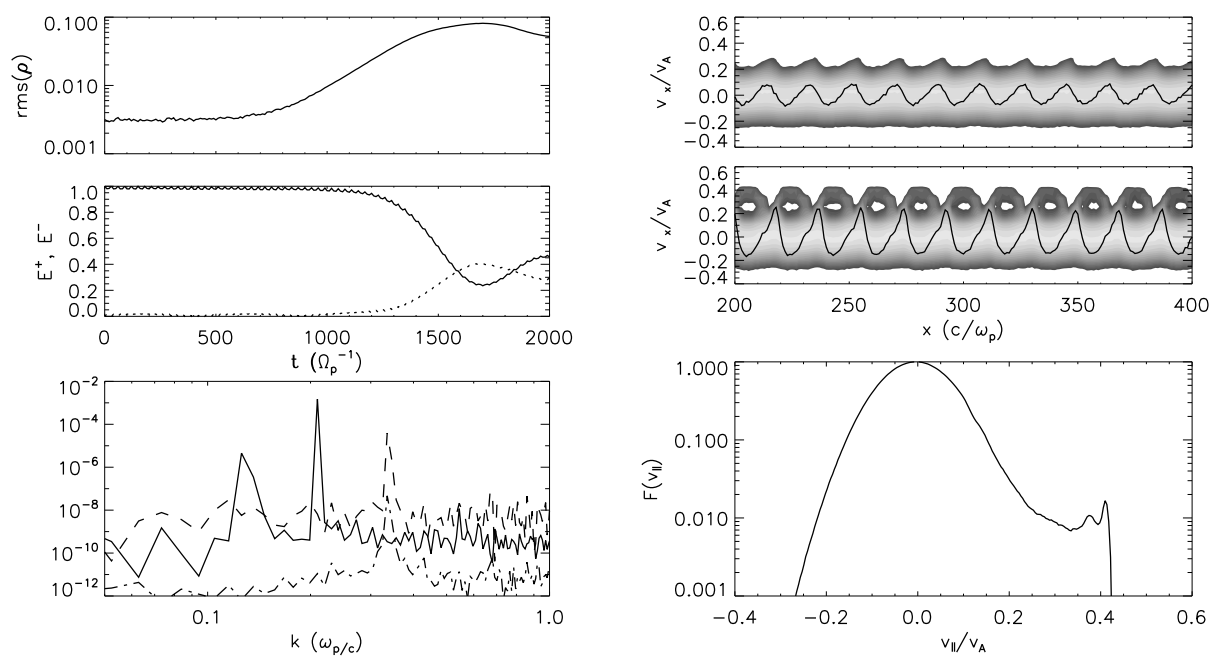

FIGURE 1. Parametric decay of a mother right handed Alfvén wave with $k=0.2 \omega_{p} / c$. Left panels report: top, the temporal evolution of density rms; middle, energy of forward (solid) and backward (dotted) waves; bottom, spectrum of magnetic (solid), density (dashed) and parallel electric field (dot-dashed) fluctuations. Right panels: top and middle, proton distribution in a portion of the phase space $x-v_{x}$ parallel to the magnetic field, at time $t=1200 \Omega_{p}^{-1}$ and $t=1500 \Omega_{p}^{-1}$ respectively; grey scale encodes the number of particles increasing from darker grey, solid line refers to the density fluctuations profile. Bottom, reduced parallel proton distribution at time $t=1800 \Omega_{p}^{-1}$.

\section{PARAMETRIC DECAY AND PROTON ACCELERATION}

Recent studies $[9,10]$ show that also wave-wave couplings leading to the generation of ion-acoustic modes, can importantly influence the ion evolution. In particular parametric instabilities of Alfvén waves produce density fluctuations which can enter in resonance with protons; these result then accelerated and some velocity beams can be generated in the distribution function.

Figure 1 reports an example of a decay instability performed with a hybrid numerical code. The mother wave is a monochromatic right-handed Alfvén wave of amplitude $\delta B / B_{0}=0.05$ and with $k=0.2 \omega_{p} / c\left(\omega_{p}\right.$ is the proton plasma frequency); the plasma betas are $\beta_{p}=0.01$ and $\beta_{e}=0.1$. The simulation box is long $L=n_{x} \Delta_{x}=600 \omega_{p} / c$, with $\Delta_{x}=1$ and $2 \cdot 10^{4} \mathrm{ppc}$. The different panels report, at left, the main properties of the instability and, at right, the corresponding evolution of the proton distribution.

The time evolution of the density rms, reported in the top left panel, shows an increase of fluctuations after $t=$ $600 \Omega_{p}^{-1}$. The growth of such fluctuations, due to the generation of compressive ion-acoustic modes excited by the instability, is accompanied with a decrease of the energy carried by the mother Alfvén wave $\left(E^{+}\right.$, solid line) and an increase of the energy of a daughter backward propagating Alfvén mode ( $E^{-}$dotted). This is a clear signature of a parametric instability of the Alfvén wave [e.g., 11]. The spectral analysis of the fluctuations reported in the lowest left panel, reveals a three-waves coupling between the mother wave (energy peak at $k_{0}=0.2$ in the magnetic energy), the secondary mode (peak $k_{r} \sim 0.13$ in the magnetic energy), and the compressive mode (peak $k_{s} \sim 0.33$ in the density and electric fluctuations). Such three-wave coupling exciting a compressive mode with $k_{s}>k_{0}$, is the typical signature of the decay instability [12].

The saturation of the instability is reached via particle trapping. Top and middle right panels of Figure 1 report the phase-space $x-v_{x}$ distribution of protons at 2 different instances, during the linear and saturation phase respectively. The presence of vortices in the phase space in the saturation stage is a clear indication of particle trapping. The vortices are strictly correlated with the density fluctuations (highlighted in the figure with the solid line, in arbitrary units), showing that protons are interacting with the parallel electric field carried by the ion-acoustic mode. A net acceleration is produced for the resonant protons and a velocity beam in the ion distribution function is hence produced (bottom right panel of Figure 1). This evolution is analogous to the dynamics found by [9] for the modulational instability.

We then suggest that parametric decay can be a possible mechanism for beam generation in the solar wind, in particular at low betas when the associated proton acceleration results more efficient [10]. Parametric instabilities play a role also in the simulations described in the next section, where the Alfvén waves evolution and the associated cyclotron heating on protons are investigated within an expanding framework. 


\section{HYBRID EXPANDING SIMULATIONS}

We have performed simulations using the Hybrid Expanding Box (HEB) model [13, 14] which describes a Cartesian approximation of a small box of plasma moving along the radial coordinate $r(t)=r(0)\left(1+t / t_{e}\right)$, here aligned with the $x$ axis, with constant velocity $U_{0}$ and a characteristic expansion time $t_{e}=r(0) / U_{0}$. Following [13] and [15], we have taken $t_{e}=2 \cdot 10^{3} \Omega_{p}^{-1}$; for computational reasons this is slightly smaller than what expected in the real solar wind at $\sim 1 \mathrm{AU}$, where $t_{e} \sim 10^{4} \Omega_{p}^{-1}$.

In order to study the proton-cyclotron heating we have introduced a spectrum of left handed Alfvén waves in the simulation domain. As in previous studies, we consider the frequency sweeping mechanism: the initial waves are relatively far from the resonance and, as the system expands, the waves become resonant with protons. In the frequency sweeping scenario the minor ions are the first species becoming resonant, then alpha particles, and finally protons. As shown by [15] the minor ions (in their case $O^{5+}$ ) absorb only a limited amount of available fluctuating energy. Then how much energy is transferred to protons depends on alphas; these can be importantly accelerated and heated by the waves, and it is not clear how much then the proton heating can be efficient. Anyway [16] and [7] have shown that in the presence of a sufficiently large velocity drift between protons and alphas, protons reach the cyclotron resonance before than the alphas; it is then the protons which exhibit the stronger scattering. For this reason, as we are interested in simulating the fast solar wind at $\sim 0.3 \mathrm{AU}$ where an important drift velocity between protons and alphas is observed, we assume that only protons interact with the waves and no other particles are included in the simulation.

We initialize the spectrum with 30 modes corresponding to wave-vectors in the range $k=0.01 \div 0.16$; in agreement with the cited previous studies, and with the in situ observations these are taken with an amplitude of $\delta B / B_{0}=3 \cdot 10^{-2}$. The simulation box is long $1200 c / \omega_{p}$ with a spatial resolution $\Delta x=1$; we use $2 \cdot 10^{4}$ particles per cell in order to well reproduce the distribution function shape. The range of the initial modes is such that the system enters in resonance after a certain time, and is maintained resonant for the duration of the simulation, as the spectrum is enough large to cover the sweeping of the proton-cyclotron frequency with distance.

We consider an initial proton bi-Maxwellian distribution function characterized by a temperature anisotropy and parallel proton beta of $T_{\perp p} / T_{\| p}=2$ and $\beta_{\| p}=0.05$ respectively, which are values compatible with those observed in fast streams at $0.3 \mathrm{AU}$ [e.g. 5]. The electron beta is assumed to be $\beta_{e}=0.1$ and, together with the initial values of anisotropy and parallel proton beta here adopted, the linear theory predicts the system to be sta-

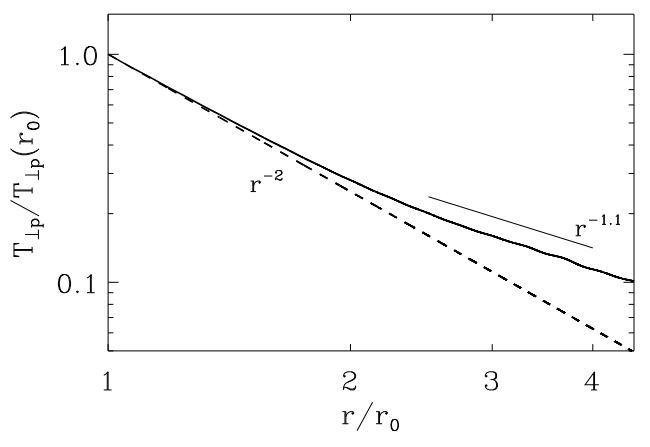

FIGURE 2. Radial dependance of the proton perpendicular temperature obtained from a hybrid expanding simulation (solid line). The slope of the adiabatic CGL prediction $r^{-2}$ (dashed line) and the observational dependence $r^{-1.1}$ inferred by Helios data between 0.3 and $1 \mathrm{AU}$ for the fast wind, are also reported.

ble to instabilities driven by the temperature anisotropy. On the contrary the theory predicts damping rates for left handed ion-cyclotron waves. So we expect that the waveparticle interaction should be dominated by wave energy losses and particle heating, once that the initial spectrum become resonant with protons.

In Fig. 2 we report the radial evolution of the perpendicular temperature. After an initial phase characterized by the CGL-adiabatic evolution and characterized by $T_{\perp p} \propto r^{-2}$, protons begin to be enter in resonance with the waves, and the temperature profile $T_{\perp}(r)$ changes its slope for larger distances. This is due to the ioncyclotron interactions that provide an increasing perpendicular heating. The resulting slope is in agreement with a power law with $\alpha=-1.1$, which is the value inferred by Helios observations [1]. The initial spectrum of waves evolve and decreases with distance, as expected in a radial expansion. However we find that it decreases faster than the WKB prediction, and this confirms that during the evolution part of the wave energy has been dissipated by the cyclotron interaction. The perpendicular heating modify also the evolution of the temperature anisotropy with respect the adiabatic expansion, providing a good agreement with the anisotropy constraints inferred by in situ observations at different distances. We compare more in detail our results with Helios and Ulysses data in another paper [17].

To show the kinetic effects of wave-particle interactions we report in figure 3 the resulting proton velocity distribution function at time $t=2000 \Omega_{p}^{-1}$. For negative parallel velocities the distribution presents a kinetic shell shape which is a typical signature of the resonant ion-cyclotron interactions. This diffuses protons in the perpendicular direction with a scattering along circles of 


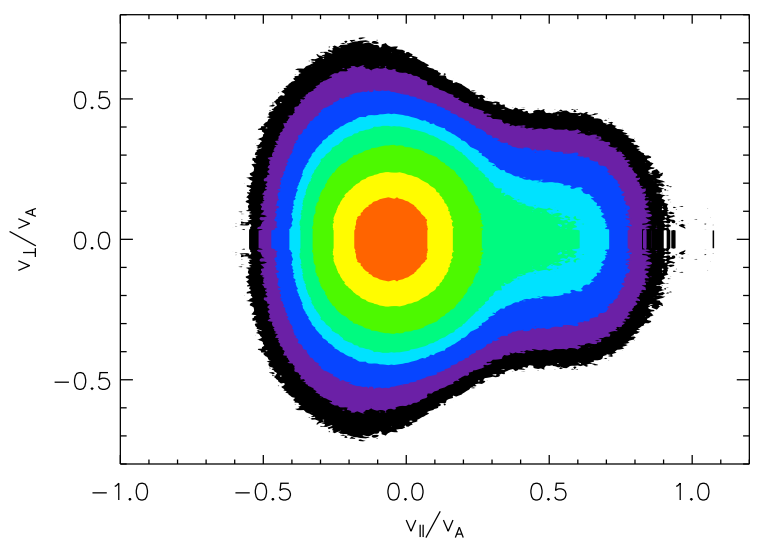

FIGURE 3. Proton distribution function in velocity space $v_{\|\|^{-}} v_{\perp}$ at time $t=2000 \Omega_{p}^{-1}$ resulting from the wave-particle interactions during the plasma expansion.

constant energy in the frame of the interacting wave; we can then observe, for $v_{\|}<0$, the increase in the perpendicular width of the distribution and the decrease in the parallel direction.

At the same time, the distribution develops also a velocity beam for $v_{\|}>0$. This is not a consequence of the ion-cyclotron interaction, which involve only protons with $v_{\|}<0$ (as we have introduced only forward propagating waves) and does not produce a parallel heating. On the contrary the acceleration of protons along the magnetic field is found to be correlated, as in the example discussed in the previous section, to parametric effects that, at the same time as the cyclotron heating, characterize the evolution of the initial Alfvén wave spectrum.

\section{CONCLUSION}

We have reported simulation results of wave-particle interactions in the solar wind plasma focusing on the kinetic processes that produce non-thermal properties in the proton distribution. First we have shown, after [9] for modulational instability, that also the parametric decay instability of Alfvén waves generating ion-acoustic fluctuations can accelerate resonant protons and produce a velocity beam along the magnetic field direction.

Then we have discussed an expanding simulation performed with the Hybrid Expanding Box model describing the evolution with distance of a spectrum of lefthanded ion-cyclotron waves interacting with solar wind protons. The model retains the main effect of a radial expansion as the adiabatic evolution for waves (WKB) and particles (CGL). At the same time kinetic effects are selfconsistently included in the simulation, so that parametric effects described above together with ion-cyclotron resonances can influence the evolution of the ion dis- tribution. It results that the perpendicular heating provided by the damping of the initial spectrum of waves becoming cyclotron resonant with increasing distance, contrast the cooling driven by the expansion and changes the slope of the proton perpendicular temperature profile in a way that is in agreement with observations. Finally the resulting proton distribution are in reasonable agreement with fast wind distribution observed in the solar wind, including ion-cyclotron heating signatures and the presence of a velocity beam along the magnetic field direction produced by parametric instabilities.

\section{ACKNOWLEDGMENTS}

This research was supported in part by ASI contract $\mathrm{n}$. I/015/07/0 "Solar System Exploration".

\section{REFERENCES}

1. E. Marsch, R. Schwenn, H. Rosenbauer, K.-H. Muehlhaeuser, W. Pilipp, and F. M. Neubauer, $J$. Geophys. Res. 87, 52-72 (1982).

2. L. Matteini, S. Landi, P. Hellinger, and M. Velli, J. Geophys. Res. 111, 10101-+ (2006).

3. J. C. Kasper, A. J. Lazarus, S. P. Gary, and A. Szabo, in AIP Conf. Proc. 679: Solar Wind Ten, 2003, pp. 538-541.

4. P. Hellinger, P. Trávnícek, J. C. Kasper, and A. J. Lazarus, Geophys. Res. Lett. 33, 9101-+ (2006).

5. L. Matteini, S. Landi, P. Hellinger, F. Pantellini, M. Maksimovic, M. Velli, B. E. Goldstein, and E. Marsch, Geophys. Res. Lett. 34, 20105-+ (2007).

6. M. Heuer, and E. Marsch, J. Geophys. Res. 112, 3102-+ (2007).

7. J. C. Kasper, A. J. Lazarus, and S. P. Gary, Physical Review Letters 101, 261103-+ (2008).

8. E. Antonucci, Space Sci. Rev. 124, 35-50 (2006).

9. J. A. Araneda, E. Marsch, and A. F.-Viñas, Physical Review Letters 100, 125003-+ (2008).

10. L. Matteini, S. Landi, M. Velli, and P. Hellinger, submitted to J. Geophys. Res. (2009).

11. L. Del Zanna, M. Velli, and P. Londrillo, $A \& A$ 367, 705-718 (2001).

12. M. L. Goldstein, ApJ 219, 700-704 (1978).

13. P. C. Liewer, M. Velli, and B. E. Goldstein, J. Geophys. Res. 106, 29261-29282 (2001).

14. P. Hellinger, P. Trávníček, A. Mangeney, and R. Grappin, Geophys. Res. Lett. 30 (2003).

15. P. Hellinger, M. Velli, P. Trávníček, S. P. Gary, B. E. Goldstein, and P. C. Liewer, J. Geophys. Res. 110, 12109-+ (2005).

16. S. P. Gary, L. Yin, and D. Winske, J. Geophys. Res. 111, 6105-+ (2006).

17. L. Matteini, S. Landi, M. Velli, and P. Hellinger, in prep. (2009). 